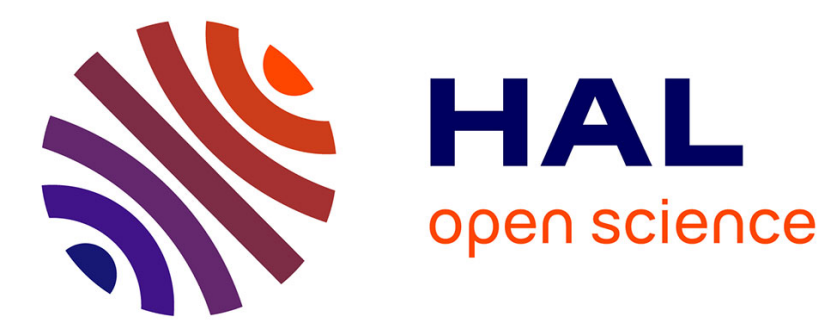

\title{
L'agriculture dans la République du Soudan
}

Marc Lavergne

\section{To cite this version:}

Marc Lavergne. L'agriculture dans la République du Soudan. Les Cahiers d'Outre-Mer. Revue de géographie de Bordeaux, 1989, 42 (167), pp.293-317. 10.3406/caoum.1989.3312 . halshs-02116887

\section{HAL Id: halshs-02116887 https://shs.hal.science/halshs-02116887}

Submitted on 1 May 2019

HAL is a multi-disciplinary open access archive for the deposit and dissemination of scientific research documents, whether they are published or not. The documents may come from teaching and research institutions in France or abroad, or from public or private research centers.
L'archive ouverte pluridisciplinaire HAL, est destinée au dépôt et à la diffusion de documents scientifiques de niveau recherche, publiés ou non, émanant des établissements d'enseignement et de recherche français ou étrangers, des laboratoires publics ou privés.

\section{(a)(1) $\$$}

Distributed under a Creative Commons Attribution - NonCommercial - NoDerivatives 44.0 


\title{
L'agriculture dans la République du Soudan
}

\section{Marc Lavergne}

\begin{abstract}
Agriculture in the Republic of Sudan. The Sudan is afflicted with chronic famines that are all the more terrifying in that its population is expanding at a rapid rate. The nation, however, could feed more inhabitants if it were better exploited. The author presents the satisfactory results obtained everywhere by peasants who have adopted agricultural techniques that are adapted to the natural settings. He analyzes the success obtained and also the disappointments encountered in the irrigation practiced on the large mechanized farms that are run by the Government. In addition, he describes the disastrous consequences of introducing mechanized farming on the vast expanses of the eastern part of the country, which is subject to scant rainfall.
\end{abstract}

\section{Résumé}

Le Soudan est affecté par des famines d'autant plus redoutables que sa population s'accroît à un rythme rapide. Le pays pourrait cependant nourrir plus d'habitants s'il était mieux mis en valeur. L'auteur met en lumière les résultats obtenus partout où des paysanneries ont adopté des techniques agraires adaptées au milieu naturel ; il analyse les réussites et aussi les limites de l'agriculture irriguée pratiquée dans de grandes fermes mécanisées gérées par l'Etat ; il décrit les conséquences désastreuses de l'introduction d'une culture mécanisée sous pluie, sur de vastes territoires de l'est du pays.

\section{Citer ce document / Cite this document :}

Lavergne Marc. L'agriculture dans la République du Soudan. In: Cahiers d'outre-mer. N 167 - 42 e année, Juillet-septembre 1989. pp. 293-317;

doi : https://doi.org/10.3406/caoum.1989.3312

https://www.persee.fr/doc/caoum_0373-5834_1989_num_42_167_3312

Fichier pdf généré le 23/04/2018 


\title{
L'agriculture dans la République du Soudan
}

\author{
par Marc-Etienne LAVERGNE*
}

\begin{abstract}
Résumé - Le Soudan est affecté par des famines d'autant plus redoutables que sa population s'accroît à un rythme rapide. Le pays pourrait cependant nourrir plus d'habitants s'il était micux mis en valeur. L'auteur met en lumière les résultats obtenus partout où des paysanneries ont adopté des techniques agraires adaptées au milieu naturel ; il analyse les réussites et aussi les limites de l'agriculture irrigucee pratiquée dans de grandes fermes mécanisćes gérées par l'Etat; il décrit les conséquences désastreuses de l'introduction d'une culture mécanisée sous pluie, sur de vastes territoires de l'est du pays.
\end{abstract}

Summary - Agriculture in the Republic of Sudan. The Sudan is afflicted with chronic famines that are all the more terrifying in that its population is expanding at a rapid rate. The nation, however, could feed more inhabitants if it were better exploited. The author presents the satisfactory results obtained everywhere by peasants who have adopted agricultural techniques that are adapted to the natural settings. He analyzes the success obtained and also the disappointments encountered in the irrigation practiced on the large mechanized farms that are run by the Government. In addition, he describes the disastrous consequences of introducing mechanized farming on the vast expanses of the eastern part of the country, which is subject to scant rainfall.

Mots-clés - Soudan; agriculture pluviale ; agriculture irriguée ; élevage ; mécanisation agricole ; agriculture traditionnelle ; grande exploitation ; métayage. 
Présenté à partir du milieu des années 70 comme le futur grenier du monde arabe, le Soudan a été frappé depuis 1983 par une terrible famine qui a fait environ 300000 victimes.

Mal connu en France, cet immense pays (le plus grand d'Afrique et du monde arabe avec plus de 2,5 millions de $\mathrm{km}^{2}$ ) peuple de 24 millions d'habitants dispose d'un potentiel agricole indéniable. Il doit ce privilège d'une part aux pluies d'été qui tombent sur une grande partie de son territoire, d'autre part à la traversée du pays par le cours moyen du Nil et ses affluents. Mais le secteur agricole, vital pour l'économie nationale (65\% des emplois, $37 \%$ du PIB et $95 \%$ des exportations), souffre depuis l'indépendance de l'absence de politique de développement économique et social.

\section{I - Le milieu et les hommes}

\section{Le milieu}

Le pays s'étend du nord au sud entre $3^{\circ} 30$ et $22^{\circ}$ de latitude nord. Le climat, désertique entre $18^{\circ}$ et $22^{\circ}$ de latitude, s'humidifie ensuite progressivement jusqu'au climat subéquatorial des marges du Zaïre. Les pluies d'été fournissent en moyenne de $3 \mathrm{~mm}$ au nord à $1500 \mathrm{~mm}$ au sud; la moitié méridionale reçoit plus de $300 \mathrm{~mm}$ de précipitations annuelles.

Le Soudan est l'un des pays les plus chauds du globe (le maximum absolu a été enregistré a Wadi Halfa, à l'extrême nord : 52 ${ }^{\circ} 5$ ); la température moyenne annuelle varie entre $20^{\circ}$ a l'extrême sud et $32^{\circ}$ au nord, les trois quarts du pays étant soumis à une température moyenne annuelle supérieure à $28^{\circ}$.

Cette gradation climatique en latitude n'est pratiquement pas influencée par le relief. En effet, $75 \%$ de la superficie sont occupés par des plaines et des plateaux étagés entre 300 et $500 \mathrm{~m}$ d'altitude (fig. 1).

La structure géologique est très simple : trois formations couvrent $92 \%$ de la surface : le socle précambrien et cambrien ( $49 \%)$, les grès nubiens $(28 \%)$ et la formation d'Oum Rouwaba (15\%).

Le socle est recouvert au Kordofan et a l'ouest par des dunes de sable fossiles appelés goz; à l'est (plaine du Butana), son érosion a donné de vastes plaines argileuses. Les grès nubiens, jurassiques ou crétacés, affleurent en discordance sur le socle au nord-ouest, une langue s'avançant au sud-est jusqu'à la Gezira. Enfin la formation d'Oum Rouwaba part du sud-est du pays en direction du nord-ouest, enserrant les monts Nuba ; elle est constituée de sables et d'argiles tertiaires.

Les montagnes, rares et isolées, sont soit des groupes d'inselbergs précambriens et cambriens ( $1500 \mathrm{~m}$ dans les monts Nuba, $2780 \mathrm{~m}$ dans les 
montagnes de la Mer Rouge : Red Sea Hills), soit des massifs peripheriques tertiaires, parfois marqués par le volcanisme (3042 m au Jebel Marra à l'ouest, $3157 \mathrm{~m}$ dans les monts Imatong au sud).
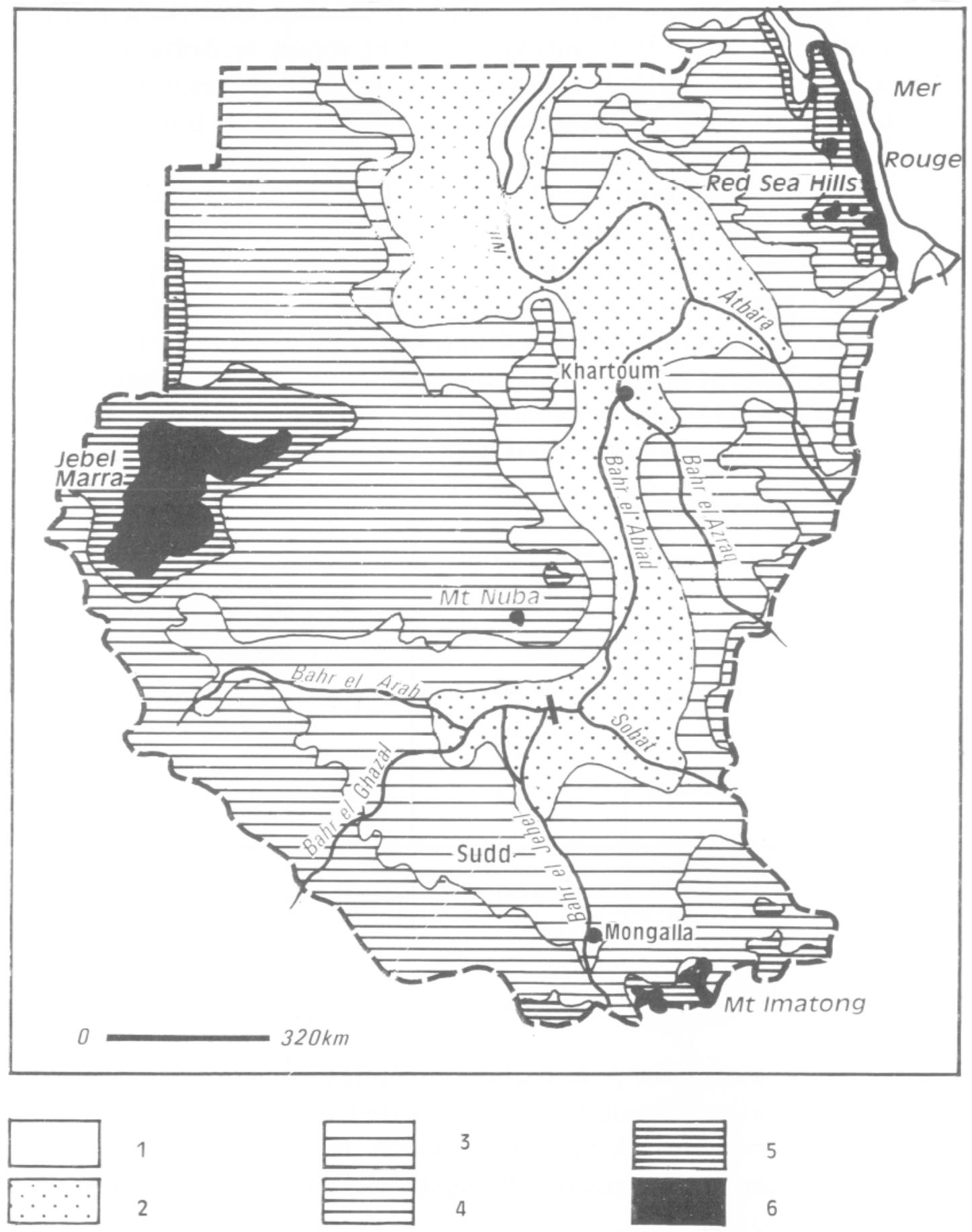

Fig. 1 - Le milieu physique

1. Moins de $300 \mathrm{~m}-2.300$ à $400 \mathrm{~m}-3.400$ à $500 \mathrm{~m}-4.500$ à $800 \mathrm{~m}-5.800$ à $1200 \mathrm{~m}$.

Le Soudan est traversé par le cours moyen du Nil et ses affluents : c'est là un facteur essentiel du potenticl agricole. Le Bahr el Jebel, ou fleuve des montagnes, pénètre au Soudan par une série de rapides pour s'assagir à 
Mongalla ; il s'étale dès lors dans toutes les directions, submergeant une vaste cuvette où 14 milliards de $\mathrm{m}^{3}$, soit $50 \%$ du débit, s'évaporent chaque année. Après $600 \mathrm{~km}$ de cours divaguant et encombré d'une végétation aquatique très dense, le fleuve reçoit sur sa gauche le Bahr el Ghazal. Il devient alors le Bahr el Abiad (ou Nil Blanc), bifurque vers l'est et reçoit le Sobat descendu des montagnes d'Ethiopie ; il toume alors vers le nord et est rejoint par le Bahr el Azraq (ou Nil Bleu), né en Ethiopie à $1480 \mathrm{~m}$ d'altitude, et parvenu au Soudan au terme d'une descente vertigineuse.

A partir de Khartoum, le fleuve est barré de six «cataractes», défilés rocheux encombrés d'îles ; il ne reçoit qu'un affluent, l'Atbara, qui roule ses eaux durant la saison des pluies, se réduisant ensuite à un chapelet de mares.

Le débit annuel du Nil à la sortie du Soudan est évalué à 84 milliards de $\mathrm{m}^{3}$ mais est extrêmement variable d'une année à l'autre : 150 milliards de $\mathrm{m}^{3}$ en 1978, 42 seulement en 1913. En outre, $80 \%$ du débit s'écoulent d'aoât à octobre, fourni par les pluies tropicales d'été sur les hauts plateaux éthiopiens, la contribution de chaque affluent au débit total est la suivante (Tab. I) :

\begin{tabular}{|l|c|c|}
\hline Cours d'eau & $\begin{array}{c}\text { \% du débit } \\
\text { annuel }\end{array}$ & $\begin{array}{c}\text { \% du débit de } \\
\text { hautes eaux }\end{array}$ \\
\hline Bahr el Azraq & 59 & 68 \\
Sobat & 14 & 5 \\
Atbara & 13 & 22 \\
Bahr el Abiad & 14 & 5 \\
\hline \multicolumn{1}{|c|}{ Total } & 100 & 100 \\
\hline
\end{tabular}

Tableau I - Contributions de ses affluents au débit du Nil

Une infime part de ce potentiel est exploitée depuis l'Antiquité, grâce à des moyens d'élévation manuelle ou animale de l'eau du fleuve, ou par la mise en culture des terres de décrue. A l'époque moderne, le recensement et l'exploitation de ces ressources ont été systématisés et pourraient donner lieu à de futures extensions.

\section{Les hommes}

La population soudanaise est d'origine africaine; elle est mêlée dans le nord d'apports arabes datant des origines de l'Islam. Bien qu'entièrement islamisée au nord du $12 \mathrm{e}$ parallelle, elle n'est que partiellement arabophone : 
d'importants groupes périphériques demeurent fidèles à leurs langues vernaculaires, Four à l'oucst, Nubiens au nord, et Béja à l'est par exemple. La cuvette du Haut-Nil et ses bordures abritent en revanche près d'une centaine de peuples nilotiques et bantous pratiquant environ 500 langues et dialectes ; réfractaires à l'arabisation et à l'islamisation, ces peuples pratiquent en majorité des cultes animistes et sont restés longtemps en marge.

Les constructions étatiques qui se sont succédées sur le territoire actuel du Soudan ne contrôlaient au début du XIXe siècle que d'une façon très lâche la population, organisée au scin de groupes tribaux autonomes : ainsi des royaumes chrétiens de Nubie avaient été anéantis à l'aube du XVIe siècle par le sultanat musulman Founj de Sennar. Celui-ci exerça jusqu'en 1821 une autorité le plus souvent indirecte sur les tribus islamisées et arabisées de la vallée du Nil, tandis qu'à l'ouest, le sultanat du Darfour s'étendait, à l'occasion, des pentes du Jebel Marra aux rives du Bahr el Abiad.

Ces deux pouvoirs reposaient sur le contrôle du commerce caravanier de 1'Egypte avec l'Abyssinie ou le bassin du lac Tchad. Les terres de pâture ou de culture demeuraient la propriété collective des tribus qui géraient, d'un commun accord, les mouvements saisonniers des troupeaux et la rotation annuclle des cultures à l'intéricur du terroir.

L'occupation turco-égypticnne (1822-1885) et la reconquête mahdiste de l'indépendance nationale (1885-1898) n'ont que peu altéré ce système, en dehors des reclassements entre tribus «collaboratrices» et tribus «résistantes», opérés pour la première fois par un pouvoir central fort.

La colonisation britannique (1898-1956) s'est attachée à développer la culture du coton à fibre longue sur de vastes périmètres irrigués. L'Etat pour mener à bien ses entreprises de misc en valeur intervient donc en louant ou achetant des terres dans la vallée, la plaine de la Gezira entre les Bahr el Azraq et Bahr el Abiad, les deltas orientaux du Gash et de Tokar. Il se mit aussi à exproprier et distribuer des domaines de culture pluviale au grè des nécessités politiques, en particulier vis-à-vis des grandes confréries religieuses.

Mais c'est sculement le Land Allotment Act de 1970, longtemps après l'indépendance, qui mit fin à la fiction de la propriété éminente des tribus sur leurs terres ancestrales. Elles conservent néanmoins l'usufruit de leurs terroirs, dont elles peuvent être dépossédécs par l'Etat pour faire place à l'extension de l'agriculture pluviale mécaniséc.

Trois types d'agriculture coexistent donc aujourd'hui au Soudan, sans entretenir de relations entre clles, et sans que l'Etat arrive à ćtablir une politique agricole cohérente (fig. 2).

Les modes de vic ruraux traditionnels, fondés sur l'association d'une agriculture céréalière pluviale et de l'élevage concernent encore $60 \%$ de la population soudanaise, fournissant $50 \%$ de la production et $30 \%$ des exportations. 


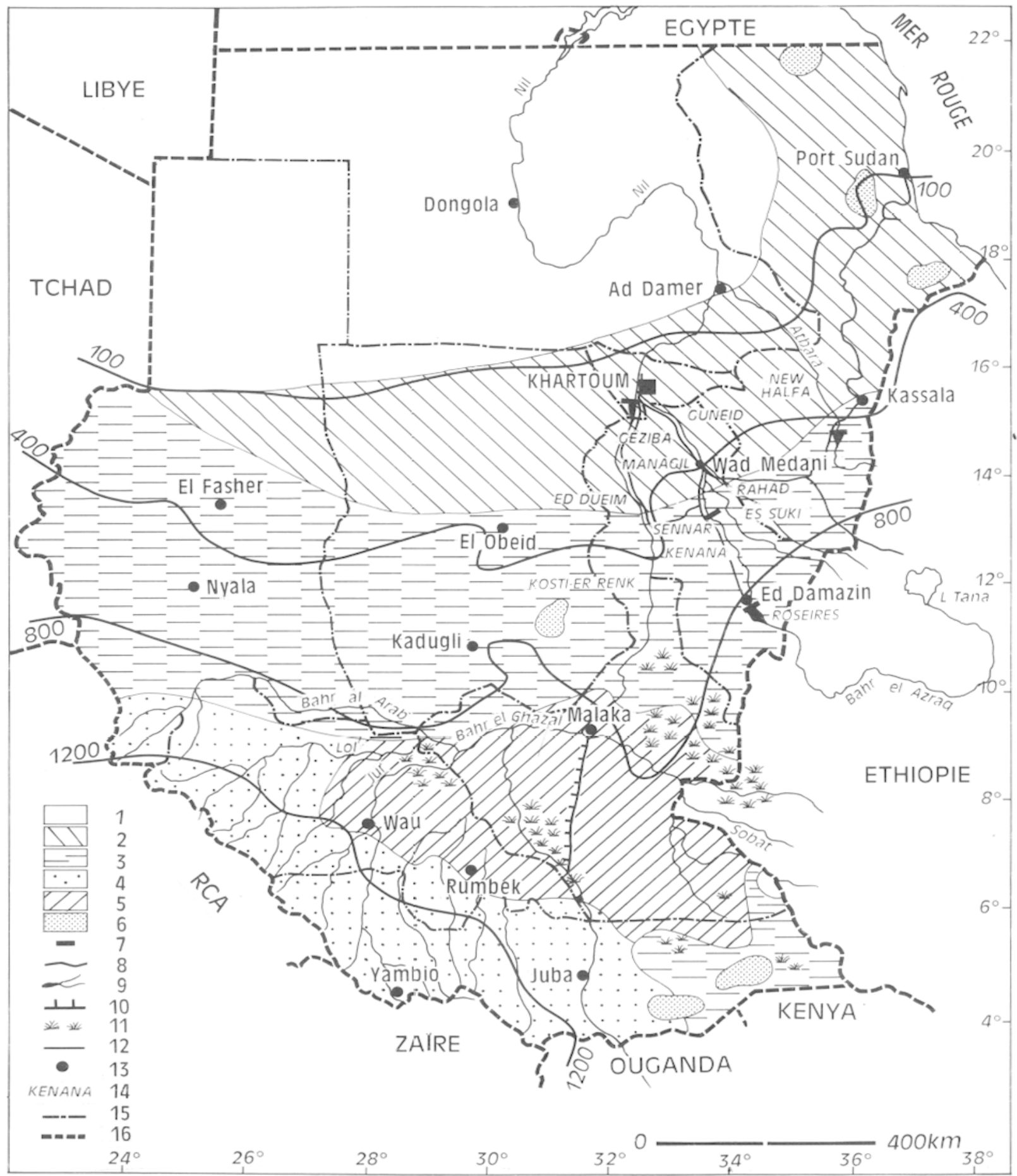

Fig. 2 - L'agriculture

1. Désertique - 2. Semi-désertique - 3. Savane arbustive - 4. Savane boisée - 5. Région inondable 6. Montagne - 7. Barrage - 8. Isohyète -9 . Rivières - 10. Canal - 11. Lit fluvial marécageux - 12. Routes principales - 13. Villes et villages - 14. Kenana : périmètre irrigué - 15. Limites régionales - 16. Frontières internationales. 
Mais l'Etat a développé au XXe siècle deux nouvelles formes d'exploitation du sol : les grandes fermes irriguécs mécanisées, destinées à la production cotonnière d'exportation; les grandes fermes de culture mécanisée sous pluie, concédées à de grands propriétaires absentéistes, et destinćes à la production spéculative de céréales et d'oléagineux.

La répartition de ces trois secteurs est officicllement la suivante : la superficie totale des terres cultivables est de 86 millions de feddan ${ }^{1}$, celle des terres cultivées de 26 millions ; sur ce demier total, 1'agriculture traditionnelle occupe 16,5 millions de feddan, les fermes irriguées 4 , les fermes mécanisécs (sous pluie) 5,5. En fait, l'étendue réelle des fermes mécanisées en culture pluviale atteint aujourd 'hui 9 millions de feddan, un tiers au moins n'ayant pas fait l'objet d'une concession officielle.

\section{II - L'agriculture traditionnelle}

1. Dans la zone désertique, en deçà de l'isohyète $250 \mathrm{~mm}$, de grandes tribus nomades continuent d'élever de vastes troupcaux de dromadaires et de petit bétail (chèvres et moutons), au cours de migrations saisonnières nord-sud. C'est le cas des quatre confédérations Béja de la province de la Mer Rouge, et de la puissante tribu des Kababich du désert libyque (fig. 3 ).

2. Dans la zone sahélo-steppique qui reçoit entre 250 et $600 \mathrm{~mm}$ de précipitations annuelles, les tribus d'éleveurs de bovins (Baggara) et de petit bétail se mêlent aux villagcois cultivant les $g o z^{2}:$ la production principale de céréales africaines (sorgho et millet) y est diversifiée par l'arachide et le sésame, et complétée par la cueillette de la gomme arabique, exsudée par Acacia senegal.

Cette agriculture est encore largement dominante dans les provinces de l'ouest, Kordofan (où elle couvre 4 millions de feddan) et Darfour. Dans les plaines argileuses de l'est, elle a considérablement reculé depuis 1945 devant l'extension de l'agriculture mécanisée.

L'agriculture sahélo-steppique est une agriculture communautaire : le cheikh de la tribu est chargé de l'attribution des parcelles à chaque famille, le travail est manuel et ne fait appel à aucun intrant industriel. Sur les sols légers de l'ouest, les jachères sont longues (5 à 7 ans), mais la pression démographique et des déficits pluviométriques contraignent à les raccourcir; la conséquence en est souvent la remise en mouvement des dunes fixées. On considère que $650000 \mathrm{~km}^{2}$ où vivent $25 \%$ de la population du Nord Soudan sont ainsi frappés par la désertification.

1. Un feddan $=0,42$ ha

2. Goz : sables dunaires fixés 
Derrière les aspects communautaires, la paysannerie de ces régions est livrée aux usuriers à travers le système du shail, avances sur les récoltes, se trouve maintenant endettée à vie. Ces terres dévastées par la disette et la famine depuis le début des années 80 constituent en fait un réservoir de main-d'oeuvre saisonnière pour les fermes irriguécs cotonnières des rives du Nil.

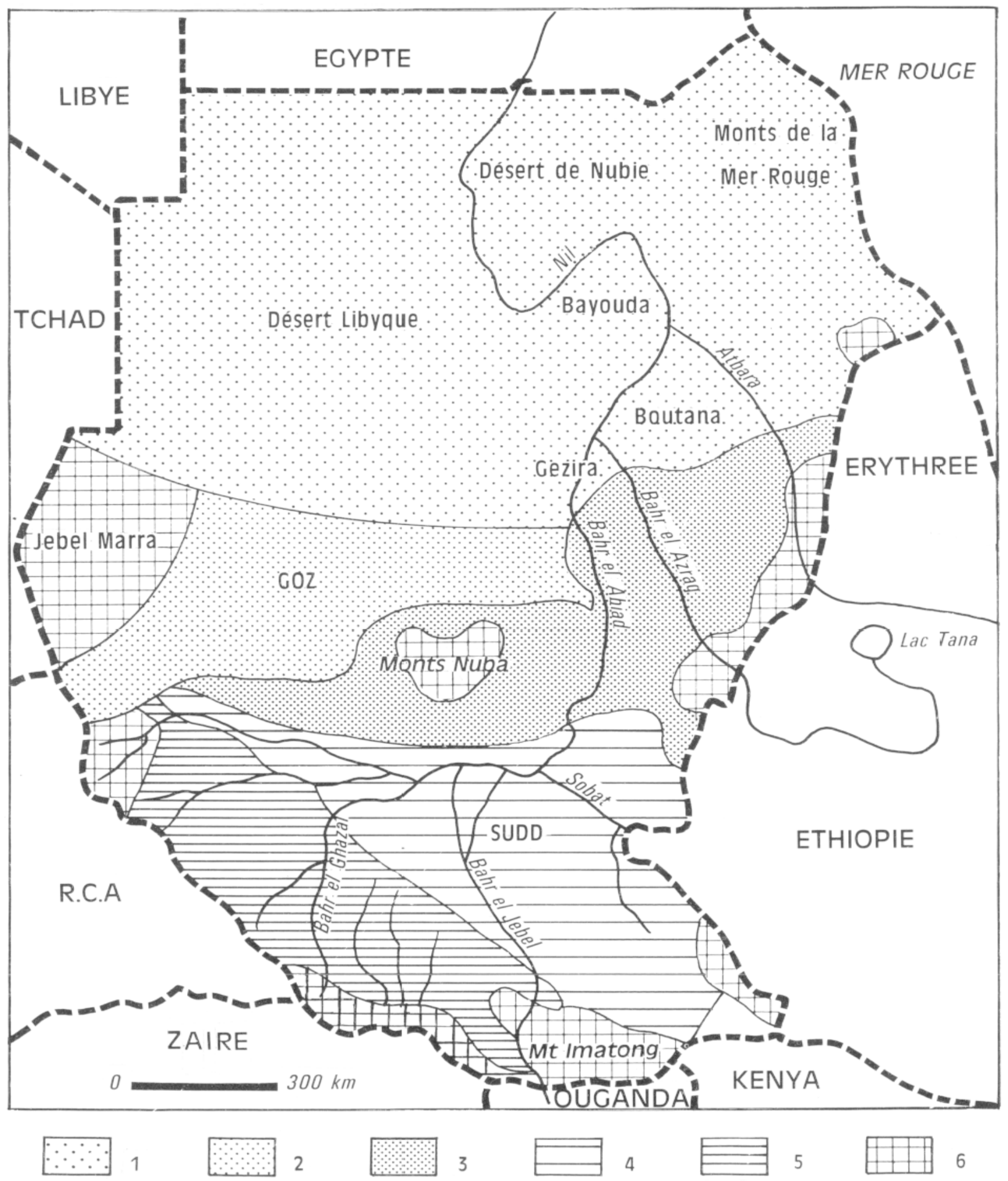

Fig. 3 - Le milieu naturel : les sols

1. Sables dunaires mobiles et sols de pédiments arides et semi-arides - 2. Sablcs dunaires fixés (goz) - 3. Sols argileux des plaines centrales - 4. Sols argileux inondables de la cuvette du IIaut-Nil - 5 . Sols ferrallitiques de climat tropical humide -6 . Sols de montagnes et collines. 
3. On peut ranger dans le secteur de l'agriculture traditionnelle les petites exploitations irriguées par pompage des rives du Nil au nord de Khartoum. Morcelées à l'extrême par le jeu des héritages, ces 230000 feddan possédés individuellement portent des palmiers-dattiers, sous lesquels sont cultivés le blé, les fèves et le sorgho ; près de Khartoum, légumes et arbres fruitiers occupent la première place.

Mais cette région, peuplée d'une paysannerie experte et active, est isolée et négligée par le pouvoir central, incapable de lui donner les moyens d'améliorer la production et de lui ouvrir des débouchés.

4. Les aires montagneuses (Jebel Marra, monts Nuba) abritent des populations purement africaines qui, profitant d'une pluviométrie plus favorable (600 à $800 \mathrm{~mm}$ ), ont su créer des civilisations agraires particulières, adaptées à leur environnement : champs en terrasses façonnées par épierrage des gros blocs de granite dans les monts Nuba, ou par la taille de banquettes dans les cendres volcaniques des versants du Jebel Marra, irrigation par détournement des torrents saisonniers, à l'aide de canalisations creusées dans le roc ou faites de troncs d'arbres évidés...

Là encore, les interventions de l'Etat sont rares et ponctuelles : fermespilotes datant du condominium, développement de la culture pluviale du coton et de la petite mécanisation dans les monts Nuba...

5. Dans la cuvette du Haut Nil occupée par les pcuples Dinka, Nuer, Shillouk, Mourlé ou Anouak, l'élevage (bovin exclusivement) redevient prédominant : l'homme s'adapte aux exigences de l'animal, transhumant entre le bord des mares pérennes et des fleuves en saison sèche et les monticules où il échappe à l'inondation et aux insectes en saison des pluies, tout en cultivant quelques champs de sorgho et de tabac. L'alimentation à base de laitages et de céréales est complétée par les produits de la chasse, de la pêche et de la cueillette.

6. Sur les collines bordant les frontières méridionales du pays, les tribus bantoues pratiquent une agriculture de clairière sur brûlis. Les plantes cultivées appartiennent au monde tropical : manioc, riz, cacaoyers, théiers, caféiers, s'ajoutent au sorgho, au tabac et aux arbres fruiticrs (manguiers).

La seule intervention de l'Etat dans cette région a été le Zandé Scheme, lancé par les Britanniques après la Scconde Guerre mondiale : ce projet devait élever le niveau de vie des planteurs par la production individuclle de coton à fibres moyennes, mais il fut rapidement victime du conflit armé entre le nord et le sud du pays.

Ce rapide tour d'horizon du sectcur agricole traditionnel laisse apparaître une société ruralc livrée à clle-même, maintenant avec de plus en plus de difficultés face à son mode de vie ancestral. La population s'accrôit, les besoins 
augmentent, mais l'Etat n'apporte aucun soutien pour diversifier l'économie, désenclaver les régions rurales, mettre sur pied un système cooperatif ou de crédit.

Par conséquent, l'exode rural gonfle les villes de nomades et de villageois déracinés et sans emploi, et se trouve amplifié par les troubles politiques et les conflits armés qui embrasent les campagnes au sud et à l'ouest.

\section{III - Les grandes fermes irriguées}

L'utilisation de l'eau fournie par les fleuves s'est développée de deux façons : exhaure grâce à de,puissantes stations de pompage, et irrigation gravitaire à partir de barrages-reservoirs.

Dès le XIXe siècle et l'occupation turco-égyptienne, la culture irriguée du coton avait été entreprise dans deux deltas intérieurs de modestes fleuves saisonniers, le Gash et le Baraka, venus d'Ethiopie. La surface cultivée y varie selon le niveau des crues canalisées vers les champs : entre 40000 et 130000 feddan pour le Gash, environ 125000 feddan pour le Baraka (Fig. 4).

L'irrigation proprement dite se rencontre le long de quatre cours d'eau : le Nil principal en aval de Khartoum ; le Bahr el Azraq (ou Nil Bleu); l'Atbara ; le Bahr el Abiad (ou Nil Blanc) et Bahr el Jebel (ou Haut Nil).

\begin{tabular}{|c|r|c|}
\hline Fleuve & Surface irriguée & $\begin{array}{c}\text { Consommation } \\
\text { milliards m3/s }\end{array}$ \\
\hline Nil principal & 352000 & 1,367 \\
Bahr el Azraq & 2850000 & 10,673 \\
Atbara & 450000 & 1,795 \\
Bahr el Abiad & 502500 & 2,116 \\
Barh el Jebel & & \\
\hline & & 15,951 \\
\hline Total & 4142500 & \\
\hline
\end{tabular}

Tableau II - Répartition des terres irriguées en $1977 / 78$

Ces terres irriguées sont en grande majorité gérées par l'Etat à travers des sociétés agricoles dépendant directement du Ministère de 1'Agriculture. Les paysans ont un statut de métayers un peu particulier, puisqu'ils sont généralement associés de façon théorique à la gestion, et astreints à des cultures obligatoires (coton) dont la commercialisation est assuree par l'Etat. 

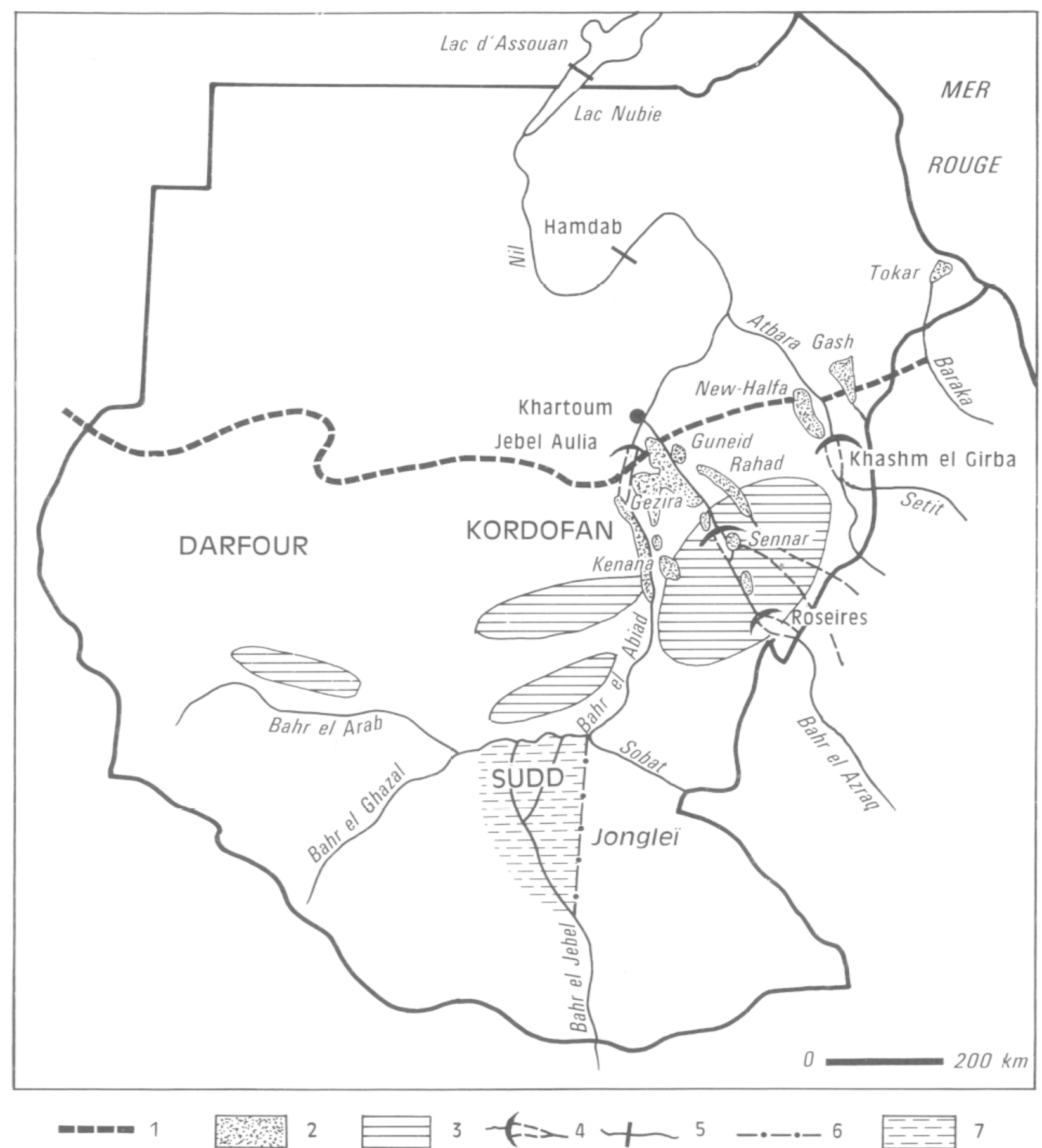

$3-f=>4$

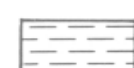

7

Fig. 4 - L'agriculture «moderne» pluviale et irriguée

1. Limite nord de la culture pluviale - 2. Périmètres irrigués - 3. Zone de culture pluviale mécanisée 4. Barrage-réservoir - 5. Barrage en projet - 6. Canal en construction - 7. Marais.

Ces fermes où le travail est très mécanisé connaissent des problèmes dus à leur gestion bureaucratique, au manque d'incitation financière pour les paysans, et au vieillissement des infrastructures. Des plans de réhabilitation sont préparés et mis en oeuvre depuis le début des années 80 , et une réforme fondamentale remplace désormais le partage du produit du coton après 
déduction des charges communes par un droit sur la terre ct l'eau, variable selon les fermes et les cultures : l'agricultcur peut ainsi savoir en début de campagne quel bénéfice attendre de ses efforts.

On peut distinguer trois secteurs géographiques :

1. Le Nil principal. C'est là que l'irrigation moderne a été introduite en 1904. Aujourd'hui, l'Etat possède six fermes de 50000 feddan au total, employant 16000 métayers dépourvus de tout soutien technique.

2. Le Bahr el Azraq (ou Nil Bleu). C'est le coeur de l'agricultu:e étatique soudanaise, avec $75 \%$ des surfaces irriguées du pays. Sur les 84 milliards de $\mathrm{m}^{3}$ roulés annuellement par le Nil, la part du Soudan a été fixéc à 18,5 milliards de $\mathrm{m}^{3}$, contre 55,5 milliards à l'Egypte et 10 réscrvés à l'évaporation à partir du réservoir du haut Barrage d'Assouan (accord soudano-égyptien de 1959).

La part soudanaise est prélevée essentiellement sur le Bahr el Azraq en période de crue, grâce à deux barrages-réservoirs : le barrage de Sennar, élevé en 1925, qui a une réserve utile de 0,7 milliard de $\mathrm{m}^{3}$, et scrt à irriguer par gravité le périmètre de la Gezira et de Managil ; le barrage de Roseires, construit en 1966 en amont de celui de Sennar qui a une capacité de stockage de 2,4 milliards de $\mathrm{m}^{3}$ à $l^{\prime}$ heure actuelle.

a) La ferme de la Gezira et son extension de Managil s'étendent entre les Bahr el Azraq et Bahr el Abiad ; cet énorme ensemble (2 millions de feddan) est géré par une seule organisation, le Sudan Gezira Board, associé à l'Etat et aux 107000 métayers. L'Etat cst le maître d'ocuvre du projet, dont il a édifié toutes les infrastructures : barrage de Sennar, canaux, voies ferrées, bâtiments ; il a également acquis ou loué la terre distribuéc aux métayers à raison de 15 ou 20 feddan à Managil ou dans la Gezira. Le Sudan Gezira Board, nationalisé en 1950, est responsable de la production, du traitement et de la commercialisation du coton à fibres extra-longues.

Enfin les métaycrs sont chargés de toutes les opérations culturales manuclles. Ils font appel aujourd'hui a une abondante main-d'oeuvre permanente ou saisonnic̀re ( 550000 chaque annéc pour la récolte du coton).

La culture du coton est obligatoire et strictement organisée. L'assolement se présente schématiquement ainsi ; dans la Gezira le coton occupe le quart de la surface, l'arachide, le blé et la jachère chacun des trois autres quarts.

Le sorgho remplace souvent l'arachide, et l'on rencontre aussi des champs consacrés aux fourrages ou aux légumes.

Les difficultés évoquees plus haut se posent ici avec une particulière acuité, aggravécs par le gigantisme de l'exploitation et son âge déjà grand, à quoi s'ajoute la commercialisation parfois difficile du coton soudanais sur les marchés extéricurs. L'essenticl demcure un état d'esprit démotivé, tant chez les 
fonctionnaires du Sudan Gezira Board que chez les métayers, qui constituent une caste de rentiers de la terre au-dessus de la masse des salariés sans statut ni protection.

Les rendements s'élèvent depuis la mise en oeuvre en 1980 d'un plan de réhabilitation financé par la Banque Mondiale, et des réformes du mode de partage de la récolte ; mais la Gezira est encore loin d'atteindre l'équilibre économique et social qu'elle pourrait offrir après 60 ans d'existence.

b) La ferme de Rahad a été conçue en 1965 pour utiliser l'eau emmagasinée dans le réservoir de Roseircs, en irriguant une bande de terres fertiles à l'cst de la rivière Rahad. Le projet n'a finalement vu le jour qu'cn 1977/78, lors de l'euphorie du mythe du Soudan "grenier du monde arabe»: le financement en a été fourni largement par des fonds de pays arabes pétroliers.

Le projet était destiné à la production de coton à fibres moyennes, d'arachides et de légumes sur des exploitations de 24 feddan, selon une rotation biennale où se succèdent coton et arachides. En réalité, le paysan tend ici aussi à assurer sa sécurité alimentaire en faisant une large place au sorgho : 90000 feddan en 1985-86, contre 118000 de coton et seulement 40000 d'arachides.

Le projet en est resté jusqu'à maintenant à sa première phase, soit 300000 feddan sur les 800000 prévus au total. La plupart des activités sont mécanisées, bien que la ferme occupe 14000 métayers ct 90000 ouvriers saisonniers.

3. L'exploitation des eaux de l'Atbara est licé à l'édification du haut barrage d'Assouan et à la nécessité de reloger la population nubienne déplacée lors de la mise en cau du réservoir. Un barrage fut construit à Khashm el Girba de 1961 à 1964 ; sa capacité était à l'origine de 1,3 milliard de $\mathrm{m}^{3}$, mais la sédimentation la réduit chaque année de 50 millions de $\mathrm{m}^{3}$.

La ferme de New Halfa couvre 450000 feddan, dont 275000 ont accueilli 50000 Nubiens ; 125000 ont été distribués aux nomades Shoukriya des environs, et 33000 sont consacrés directement par l'Etat à la canne à sucre. La réussite de cette ferme d'Etat est inégale : si les Nubiens sont d'excellents agriculteurs, il n'en est pas de même des nomades récemment sédentarisés, qui préfèrent consacrer lcurs efforts à l'ćlevage, pourtant sévèrement limité à l'intéricur du périmètre de la ferme.

\section{Le Bahr el Abiad et le Bahr el Jebel. Les rives du Bahrel Abiad (ou Nil Blanc)} ont êté progressivement équipécs de pompes à moteur par des entrepreneurs individuels, après la construction du barrage du Jebcl Aulia en 1929. Les 360 petites fermes ont été nationalisécs en 1970 et réunies dans la White Nile Agricutural Corporation. Mais cette entreprise ne dispose pas des moyens nécessaires pour entretenir et rentabiliser un domaine aussi morcelé ; la privatisation en a donc été décidée en 1986, mais selon des modalités qui demeurent confuses. 
A la hauteur de Kosti, sur la rive droite du fleuve, a été implanté le fameux projet sucrier de Kenana, qui avec 81000 feddan irrigués, devait symboliser la capacité du Soudan à détrôner Cuba comme premier producteur mondial. Conçu en 1972, lors d'une période d'extrême tension du marché, Kenana est entré en production en 1980, après le retournement de la conjoncture. Entre temps, le coût global du projet ćtait passé de 190 millions à 1 milliard de dollars US. Aujoud'hui, Kenana produit 300000 tonnes de sucre par an et témoigne d'une indiscutable réussite technique. Mais la production couvre à peine la consommation intérieure, et le coût des 80 techniciens expatriés grève la rentabilité de l'ensemble.

De grands espoirs d'extension de l'agriculture irriguée avaient été placés dans les travaux d'aménagement de la cuvette du Bahr el Jebel (ou Haut $\mathrm{Nil})$ : on évalue à 45 milliards de $\mathrm{m}^{3} \mathrm{lc}$ volume de l'eau qui s'y évapore, dont 20 pourraicnt être récupérés.

Le premier projet était celui du canal de Jongleï, qui devait permettre d'augmenter le débit du Bahr el Abiad de 4,75 milliards de $\mathrm{m}^{3}$ par an à Malakal, à partager également entre l'Egypte et le Soudan. Le déclenchement de la Dcuxième Gucrre du Sud en mai 1963 a intcrrompu les travaux de creusement terminés aux deux tiers. Il semble désormais probable qu'aucun de ces travaux d'aménagement ne reprendra avant qu'un règlement durable de la question de l'unité nationale ait été trouvé.

D'autres extensions seraient possibles à proximité du Bahr el Azraq où abondent les terres fertiles; mais les 2,5 millions de feddan irrigables recensés exigcraient 8 milliards de $\mathrm{m}^{3}$ d'eau supplémentaires, qui ne pourraient être fournis que par l'aménagement de la cuvette du Bahr cl Jebel.

Seuls demeurent donc envisagés actucllement le projet de barrageréservoir de Mérowé sur la 4e cataracte, qui pourrait démarrer en 1996, et celui de la Setit, affluent de l'Atbara. Le Soudan s'approche en effet rapidement de la plcine utilisation de sa part des eaux du Nil, tandis que l'Egypte s'inquiète de ce que le débit de celui-ci n'a atteint qu'une fois, au cours de la décennie écoulée, son nivcau moyen centenaire.

Mais le Soudan, très endetté (10 milliards de dollars de dette extérieure publique), trouvera difficilement les fonds nécessaires, tant que la situation politique ne sera pas stabiliséc par un retour à la paix civile.

C'est pour cela que s'est amplifié depuis une dizaine d'annécs le troisième volet de l'agriculture soudanaise, c'est-à-dire les grands domaines mécanisés en culture pluviale.

\section{IV - L'agriculture pluviale mécanisée}

Ce type d'exploitation est apparu après la Dcuxic̀meGuerre mondiale, au Soudan oriental (région de Gedaref). Son objet est la culture extensive 
du sorgho et d'oléagineux (sésame, toumesol) pour le marché intérieur et l'exportation.

La culture est pratiquée surde grands domaines géométriques situés dans la zone recevant de 400 a $800 \mathrm{~mm}$ de précipitations par an : province de Gedaref, sud des provinces Blue Nile et White Nile, extrême nord de la province Upper Nile et province du Southern Kordofan, soit environ 9 millions de feddan au total.

\begin{tabular}{|l|c|c|c|}
\hline \multicolumn{1}{|c|}{ Région } & $\begin{array}{c}\text { Concessions } \\
\text { officielles }\end{array}$ & $\begin{array}{c}\text { Exploitations } \\
\text { non-autorisées }\end{array}$ & Total \\
\hline El Gedaref & 3 & 3 & 6 \\
Southern Kordofan & 0,5 & 0,5 & 1 \\
Upper Nile + White Nile & 0,5 & - & 0,5 \\
Blue Nile & 1,5 & - & 1,5 \\
\hline \multicolumn{1}{|c|}{ Total } & 5,5 & 3,5 & 9 \\
\hline
\end{tabular}

Tableau III - Lesfermes mécanisées : superficie exploitée (en millions de feddan)

Ces terres sont normalement concédées par la Mechanized Farming Corporation (MEC), organisme d'Etat fondé en 1970, lorsque celui-ci s'attribua la pleine propriété des 545 millions de feddan non enregistrés du territoire national. En outre, de nombreuses appropriations «sauvages» ont eu lieu avec la complicité des autorités régionales ou locales : accaparement des terres communautaires par les chefs tribaux, qui les vendent ou les mettent euxmêmes en culture. Les lots distribués par la MEC sont de 1000 feddan au minimum, pour une duree de 25 ans ; ils sont acquis par des individus ou des sociétés, en échange d'un loyer annuel symbolique (1 LS/feddan, soit 0,60 FF).

Les plus grands domaines atteignent 200000 feddan d'un seul tenant, et sont exploités par des entreprises à capitaux mixtes associant l'Etat soudanais avec l'Egypte (dans le cadre des accords de «complémentarité» entre les deux pays), ou avec l'Arab Authority for Agricultural Development (A.A.A.I.D.) ; certaines grandes exploitations sont aussi le fait de capitalistes soudanais liés à des groupes arabes ou américains.

Après obtention de la concession, le premier stade est celui du défrichage : la steppe arborée est entièrement rasée à l'aide de puissants engins mécaniques. Le deuxième stade est celui de la mise en culture ; la saison culturale correspond à la saison des pluies, entre juin et octobre. Labours et 
semailles, entièrement mécanisés, ont lieu dès les premières averses. Puis les domaines détrempés, impraticables, sont déscrtés jusqu'à la récolte, qui a licu entre octobre et janvicr selon les cultures.

Le sorgho s'impose de loin comme la culture principale ; il peut en effet aisément être stocké, et trouver de larges débouchés à l'exportation (comme aliment pour le bétail ou matière première de l'amidon et du glucose), ou sur le marché intérieur, étant l'aliment de base dans les campagnes. Il n'exige en outre que peu de façons culturales et de main-d'ocuvre.

Les oléagineux jouissent d'une faveur grandissante ; mais le sésame est sensible aux attaques d'insectes et aux maladies, et le tournesol, récemment introduit, a besoin d'une bonne pluviométrie et d'une parfaite homogénéité de plantation. La récolte est en partie effectućc manucllement, par les paysans évincés du domaine ou par les villagcois des alentours, voirc par les réfugiés ethiopiens ou des migrants venus des provinces de l'ouest. Ensuite, la terre est laissée à nu durant six mois. Les effets négatifs de ce type d'agriculture apparaissent de plus en plus nettement; ils se font sentir à la fois sur l'environnement naturel et sur l'équilibre économique et social des régions productrices et du pays dans son ensemble : l'absence de rotation culturale et l'arrachage de toute la couverture végétale provoquent une érosion et une stérilisation rapide des sols ; ces grandes exploitations d'un seul tenant en monoculture sont très sensibles aux attaques des parasites; les variations climatiques auxquelles est soumise la production sont amplifiécs par la disparition de la couverture végétalc, qui scmble en outre ĉtre à l'origine d'un certain déssc̀chement du climat. Les conséquences sociales de ce type d'agriculture sont désastreuses : les terres accaparées par des citadins privent les paysans de leurs moyens d'existence, et les contraignent à rejoindre les cohortes des déracinćs aux abords des grandes villes. Les parcours des nomades sont entravés par les domaines qu'ils doivent contourner, et leurs pâturages se réduisent considerablement. Ainsi est détruite une économic qui fait un usage rationncl des ressources naturelles et procure un revenu et unc intégration sociale à une part importante de la population.

Les revenus des fermes mécanisées ne sont pas un instrument de développement économique du pays : a l'intérieur, la récolte du sorgho est stockée pour faire monter les cours, par les exploitants eux-mêmes ou par les banques, principalement islamiques, qui le leur achètent. Cette spéculation peut avoir des effets catastrophiques, comme lors de la famine de 1984-85 ; à l'extérieur, l'objectif des producteurs est l'obtention de devises qui ne sont pas rapatrićcs au Soudan, où clles scraient réquisitionnécs par la Banque centrale, mais placécs à l'étranger, principalement en Suisse et au Royaume-Uni. Seule une petite partie sert de monnaie d'échange dans des opérations de troc, pour l'importation d'intrants indispensables ou de biens de consommation. Dans le 
climat d'incertitude politique et économique actuel, ces revenus ne sont pas investis dans des activités productrices.

En dépit de ces critiques, la distribution de concessions agricoles a été, pour les gouvernements soudanais successifs, un moyen commode de s'assurer la fidélité de la bourgeoisie citadine et des notables religieux de la vallée du Nil.

Amenés à s'étendre toujours plus au sud, en quête de terres fertiles et d'une meilleure pluviométrie, les concessionnaires se trouvent aujourd'hui confrontés à des peuples peu disposés à se laisser spolicr, et auxquels le soutien de l'Armce de Libération du Peuple Soudanais est acquis : Ingessana et Bourou des confins éthiopiens, Dinka du Upper Nile, Nuba du Southern Kordofan.

Il n'en reste pas moins que de très vastes espaces (près de 60 millions de feddan) pourraient encore être mis en culture pluviale dans des conditions rationnelles, lorsque la paix sera revenue, et que les ressources du pays pourront être plus équitablement réparties.

Une agriculture et un élevage traditionnels livrés à eux-mêmes, préssurés par les usuriers, les grands négociants en bétail et en céréales, et les sociétés de commercialisation étatiques (gomme arabique... ; de grandes fermes irriguées gérées bureaucratiquement parl'Etat, cultivées pardes métayers auxquels toute initiative est interdite, obtenant à grand frais (machines, insecticides) des productions difficiles à ćcoulcr (coton)...; des concessions en culture pluviale mécanisée qui ne parviennent pas à assurer l'équilibre alimentaire du pays, ni à lui fournir les moyens de son développement économique : le tableau peut sembler très sombre ; il l'est certes, mais plus à cause des politiques économiques et sociales suivies que de conditions naturclles défavorables.

La variabilité des précipitations, la fragilité de la couverture végétale, sont des faits connus en Afrique subsaharienne. Le Soudan est victime du surpâturage, de la déforestation, de l'extension des cultures au-delà des limites traditionnelles, du raccourcissement des jachères qui concourrent à destabiliser la population rurale ; mais dans une large mesure, ces phénomènes sont dus à une mauvaise allocation des ressources, en faveur exclusivement des fermes irriguées et des concessions mécanisées.

La généralisation d'un crédit agricole accessible à tous, la création de coopératives, de petites industries agro-alimentaires, le désenclavement des régions rurales, amélioreraient le sort de la paysannerie, tandis que les fermes mécanisées, suréquipées, mais sous-peuplées et sous-productrices, pourraient accueillir l'excédent de population des zones septentrionales dégradées ; une gestion plus souple de ces formes permettrait de diversifier et d'augmenter la production, tout en y diminuant la consommation de machines coûteuses et souvent inadaptées. 
La réalisation de ces objectifs ne permettrait pas de faire du Soudan le "grenier du monde arabe» : à cela s'oppose l'absence de marché commun arabe, la faiblesse structurelle des cours mondiaux des produits agricoles, qui favorisent l'égoïsme sacré des partenaires possibles, et les politiques d'accroissement de la production nationale suivies jusque dans des régions désertiques de la péninsule Arabique. Au moins permettraient-elles de nourrir la population et de fournir la base d'un développement économique et social équilibré.

\section{BIBLIOGRAPHIE}

Ouvrages généraux:

TOTHILL, J.D., ed., 1948. Agriculture in the Sudan. Londres, Oxford University Press. $\mathrm{Ce}$ remarquable ouvrage permet encore de comprendre les fondements de l'agriculture soudanaise.

ZAHLAN, A.B., ed., 1984. Agricultural bibliography of Sudan 1970-1983. Selected, classified and annotated. Amman, Abdelhamid Shoman Foundation ; Londres, Ithaca Press, 312 p. + index. Un ouvrage bien conçu, indispensable pour l'étude de cette période où l'agriculture soudanaise a connu d'importants changements.

ZAHLAN, A.B., ed., 1986. The Agricultural Sector of Sudan, Policy and System Studies. Amman, Abdelhamid Shoman Foundation ; Londres, Ithaca Press. Par les meilleurs spécialistes, une actualisation de qualité à l'ouvrage précédent.

(1) EL-TOM, M.A., 1975. The rains in the Sudan. Khartoum, University Press, 48 p. + cartes et diagrammes. Travail de synthèse très utile en particulier par ses 61 annexes.

(2) WHITEMAN, A.J., 1971. Geology of the Sudan Republic. Oxford, Clarendon Press. WILLIAMS, M.A.J. et ADAMSON, D.A., ed., 1982. A Land between two Niles. Quaternary geology and biology of the central Sudan. Rotterdam, A.A. Balkema, 237 p + index. Dix études très documentées sur la formation de l'écosystème de l'entre-dcux-Nils.

(3) Sur la question des eaux du Nil, deux travaux récents font référence :

WATERBURY, J., 1979. Hydropolitics of the Nile Valley. Syracuse University Press, 254 p. + notes et index.

BECHIR, M.O., ed., 1984. The Nile Valley Countries. Continuity and Change. Khartoum, University of Khartoum, vol. 1, 176 p. ; vol. 2, 154 p.

Sur l' hydrologie du Nil Bleu, voir également :

EL-KHALIFA, M.D., ed., 1985. Blue Nile River from the Ethiopian Border to Khartoum. Khartoum, University of Khartoum, $69 \mathrm{p}$.

(4) Parmi plusieurs ouvrages fondamentaux sur l' histoire moderne et médiévale du Soudan, on peut choisir : 
HOLT, P.M. et DALY, M.W., 1979. The History of the Sudan ; from the coming of Islam to the present Day. Londres.

(5) HASHIM AWAD MOHAMED, 1971. The Evolution of Landownership in the Sudan.

The Middle East Journal, vol. $25, \mathrm{n}^{\circ} 2$ : repris dans: The Evolution of Agrarian Relations in the Sudan, A reader. La Haye, El Fatih Shaaeldin, ed., fev. 1987 (p. 31-56).

(6) Seeds Needs : Rains and Grains. Sudanow, octobre 1985, p. 25-26.

(7) Sur le mécanisme de la désertification au Soudan, il faut lire le travail exhaustif de :

IBRAHIM, F.N., 1984. Ecological Imbalance in the Sudan - with Reference to Desertification in Darfur. Bayreuth, 192 p. + index et cartes couleur h.t. et de deux Géographes suédois présentant une opinion contradictoire :

HELLDEN, U., 1984. Drought impact monitoring : a remote sensing study of desertification in Kordofan. University of Lund, $61 \mathrm{p}$.

OLLSON, K., 1985. Remote sensing for fuelwood resources and land degradation studies in Kordofan. University of Lund, $182 \mathrm{p}$.

University of Khartoum. Institute of Environmental Studies et ETMA/USAID, 1985. Gedaref District Study Area, Final report. Khartoum, 197 p. + bibl.

University of Khartoum, Institute of Environmental Studies et Government of Kordofan region, 1986. Kordofan Rehabilitation Development Strategy. Vol. 1.

Main report. Khartoum, 238 p. + bibl.

(9) Land reclamation: Gimma shelter. Sudanow, juillet 1985, p. 15-17.

(10) Pour une étude détaillée, voir les documents produits lors de la Conférence sur le développement agricole de la région nord. Ed-Damer, février 1985 (en arabe) :

- l'investissement agricole dans la région nord, 24 p. + annexes.

- la complémentarité (égypto-soudanaise), 22 p.

- les grands travaux et les services d'aide et de soutien à l'agriculture, $29 \mathrm{p}$.

- Présent et futur de l'agriculture dans la région : annexes.

Lire également :

AL-SHABI, A., 1986. Themes from Northern Sudan. Londres, 145 p. + bibl. et index. Plus particulièrement «Economic change and individual initiative, the Case of a developing community in northern Sudan», p. 91-107.

Hunting Technical Services Ltd, 1978. Agricultural Development of Jebel Marra Area, Final report. Borehamwood/Londres. Par un bureau d'études d'implantation ancienne, une étude minutieuse de ce massif mal connu.

(12) Sur les modes de vie traditionnels des peuples nilotiques, se reporter à l'ouvrage historique du célèbre anthropologue :

EVANS-PRITCHARD, E., 1935. Les Nuer. Description des modes de vie et des institutions politiques d' un peuple nilote. Trad. française. Paris, Gallimard, 1968, 301 p. + bibl. et index.

\section{Voir également :}

EL-SAMMANI, M.O., 1984. Jongleï Canal, Dynamics of planned Change in the Twic Area. Khartoum, University of Khartoum, 184 p. + bibl. 
(13) Par exemple :

RUSSEL, M. Contemporary Bari Economy in historical perspective. In : Bechir, M.O. supra (3), p. 81-99.

(14) CATFORD, J.R., 1953. The introduction of cotton as a cash crop in the Maridi area of Equatoria. Sudan Notes and Records, vol. 34, p. 153-171; repris dans : The Evolution of Agrarian Relations in the Sudan, supra (5), p. 57-88.

Zande Cotton Scheme : Back from the Dead. Sudanow, fevrier 1986, p. 21.

(15) LAVERGNE, M., 1987. Exode rural et croissance urbaine au Soudan. Bulletin du $C E D E J$, (Le Caire), $\mathrm{n}^{\circ} 22$, p. 123-138.

(16) SALIH, H.M., 1979. The agricultural development in the Gash Delta: Problems of Pastoralism and Participation. Document ronéo., University de Khartoum, DSRC, 35 p.

(17) EL-JACK, A.H., 1982. The Management of Resettlement Schemes in the Sudan. Khartoum, ESRC, $96 \mathrm{p}$.

(18) Focus on Agriculture : Backbone bends. Sudanow, mars-avril 1987, p. 34-36.

The Gezira Scheme : Making a Come back. Sudanow, juin-juillet 1987, p. 18-19.

(19) Zcidab Agricultural Scheme : still viable. Sudanow, octobre 1982, p. 41-42.

(20) La gestion des eaux du Nil Bleu est traitée en détail dans :

Blue Nile Study Consultants, 1978. Blue Nile Water Study, Phase IA, Avai'ability and Use of Blue Nile Water, vol. 1 The Main Report. Khartoum, Ministry of Irrigation and Hydroclectric Energy.

(21) La littérature concernant la ferme de la Gezira est très abondante. Lire surtout :

BARNETT, T., 1977. The Gezira Scheme, an Illusion of Development. Londres, 181 p. + bibl.

HASSAN, K.I., 1984. Production Relations in the Sudanese Agriculture, The Case of the Gezira Scheme. University de Khartoum, DSRC, 26 p.

Agriculture: New Life for Gezira. Sudanow, févricr 1985, p. 15-16. Sur la réhabilitation de la ferme et les modifications de structure opérées depuis 1980, voir également la note (18).

(22) ABDELSADIQ, A.M., 1984. Evaluation et postévaluation des projets agricoles au Soudan : le projet de Rahad. Université de Rennes, thèse de 3e cycle, $267 \mathrm{p}$.

(23) Sudan Ministry of Agriculture and Natural Resources. Agricultural Situation and Outlook, Annual report 1985-86. Khartoum, 76 p.

(24) voir note (17).

(25) Waterbury, op. cit. (3), p. 191-192.

(26) Waterbury, op. cit. (3), p. 210-241.

WATERBURY, J., 1986. Soudan : priorité au Nil Bleu ou au Nil Blanc. Bulletin du CEDEJ, $\mathrm{n}^{\circ} 19, \mathrm{p} .71-106+$ bibl.

(27) Blue Nile Study Consultants, op. cit. (20).

(28) L'agriculture pluviale mécanisée a été assez peu étudiće jusqu'à présent. Voir Gedaref District Study Area, op. cit. (8).

LAVERGNE, M. et GHEERAERT, F., 1986. La colonisation agricole dans la région de Damazin (Nil Blcu - Soudan). Bulletin du CEDEJ, $\mathrm{n}^{\circ} 19$, p. 119-132. 
SIMPSON, M.C. Large scale mechanized rainfed farming developments in the Sudan. In : Evolutions of Agrarian Relations, op. cit. (5), p. 269-289.

(29) Voir Gedaref...op. cit. p. 28-28 et 65-72.

Mechanized Farming Corporation : No rain, no harvest. Sudanow, avril 1985, p. 13-15. (30) ABDEL GHAFFAR, M.A., 1974. Shaykhs and followers. Political Struggle in the Rufa' a el Hö̈Nazirate in the Sudan. Khartoum, University Press, 161 p. + bibl. et index. Un essai passionnant sur la vie d'une tribu de pasteurs au sud du Nil Bleu.

Voir également Sudanow, art. cit. (6).

(31) MAHMOUD, F.B., 1984. Sudanese Bourgeoisie, Vinguard of Development. Khartoum, University Press, 152 p. + Appendice et bibl.

Article reçu en novembre 1987. 


\begin{tabular}{|c|c|c|c|}
\hline \multicolumn{4}{|c|}{ ANNEXES } \\
\hline \multicolumn{4}{|c|}{$\begin{array}{l}\text { Les principales productions agricoles du Soudan } \\
\text { Moyenne } 1980-81 / 1984-85\end{array}$} \\
\hline \multicolumn{4}{|l|}{ COTON } \\
\hline Région, province & $\begin{array}{l}\text { Superficie } \\
\text { milliers de feddan }\end{array}$ & $\begin{array}{l}\text { Production } \\
\text { milliers de tonnes }\end{array}$ & $\begin{array}{l}\text { Rendement } \\
\text { kg/feddan }\end{array}$ \\
\hline \multicolumn{4}{|l|}{ 1. Fibres longues } \\
\hline Gezira et Managil & 370 & 203 & 557 \\
\hline Blue Nile & 35 & 13 & 362 \\
\hline White Nile & 54 & 18 & 343 \\
\hline Tokar & 17 & 3 & 193 \\
\hline TOTAL & 496 & 242 & 487 \\
\hline \multicolumn{4}{|l|}{ 2. Fibres moyennes } \\
\hline Gezira & 102 & 80 & 783 \\
\hline Halfa el Gedida & 74 & 41 & 552 \\
\hline Suki & 26 & 19 & 500 \\
\hline Blue Nile & 21 & 12 & 581 \\
\hline White Nile & 19 & 8 & 447 \\
\hline Rahad & 117 & 80 & 682 \\
\hline Zeidab & 5 & 2 & 478 \\
\hline TOTAL & 364 & 256 & 703 \\
\hline \multicolumn{4}{|l|}{ 3. Fibres courtes } \\
\hline Monts Nuba & 73 & 10 & 137 \\
\hline Gedaref & 7 & 1 & 132 \\
\hline Blue Nile & 29 & 6 & 193 \\
\hline Equatoria & 30 & 2 & 73 \\
\hline TOTAL & 139 & 19 & 136 \\
\hline TOTAL GENERAL & 977 & 517 & 529 \\
\hline
\end{tabular}




\section{CULTURE DU SORGHO}

Région, province

Superficie Production Rendement

milliers de feddan milliers de tonnes kg/feddan

1.Irrigue

Nord

Nil

7

15

41

Gash

New Halfa

Suki

49

Gezira et Managil

White Nile

359

Blue Nile

39

51

Rahad

39

Tokar

24

TOTAL

13

416

429

169

472

$14 \quad 369$

$21 \quad 416$

$25 \quad 644$

9364

2. Pluvial mécanisé

Gedaref

Damazin

White Nile

Sud Kordofan

Sud Darfour

Province Sud

TOTAL

3. Pluvial traditionnel

Province Gezira

144

White Nile

Blue Nile

Sud Kordofan

Nord Kordofan

Sud Darfour

Nord Darfour

Province Sud

TOTAL

TOTAL GENERAL

2512

1149

240

363

1

378

4643

167

556

402

419

372

49

760

2869

8140
628

285

1187

680

269

53

87

1

97

454
34

29

133

87

46

79

5

126

539

2011
271

235

222

240

400

256

256

239

176

239

215

111

212

107

166

188

247 


\section{ARACHIDE}

Région, province

1. Irrigué

Superficie Production Rendement milliers de feddan milliers de tonnes $\mathrm{kg} /$ feddan

New Halfa

Gezira

Suki

Rahad

TOTAL

2. Pluvial traditionnel

Blue Nile

White Nile

Sud Kordofan

Nord Kordofan

Sud Darfour

Nord Darfour

Région Sud

TOTAL

TOTAL GENERAL

\section{SESAME}

Région

1. Pluvial mécanisé

Gedaref

Blue Nile

Sud Kordofan

Renk

TOTAL

2. Pluvial traditionnel

Blue Nile

White Nile

Sud Kordofan

Nord Kordofan

Nord Darfour

Sud Darfour

Région Sud

TOTAL

TOTAL GENERAL

201

30

705

138

11

178

1424

2012
37

169

12

63

272

Superficie milliers de feddan milliers de tonnes

Rendement $\mathrm{kg} / \mathrm{feddan}$

312

225

24

27

588

104

10

49

212

749

614

803

775

779

494

275

227

216

198

162

208

222

287

$\begin{array}{lll}699 & 355 & 222 \\ 981 & 545 & 287\end{array}$

110

110

132

121

119

127

107

58

94

92

$\begin{array}{lll}161 & 20 & 127\end{array}$

85 
MIL

Région, province

Nord Kordofan

Nord Darfour

Sud Darfour

TOTAL

\section{BLE*}

Région

Gezira et Managil

New Halfa

White Nile

Nil

Nord

TOTAL

$\begin{array}{ccc}\begin{array}{c}\text { Superficie } \\ \text { milliers de feddan }\end{array} & \begin{array}{c}\text { Production } \\ \text { milliers de tonnes }\end{array} & \begin{array}{c}\text { Rendement } \\ \text { kg/feddan }\end{array} \\ 1186 & 88 & 74 \\ 402 & 48 & 119 \\ 994 & 174 & 175 \\ 2886 & 363 & 125\end{array}$

Superficie Production Rendement milliers de feddan milliers de tonnes $\mathrm{kg} / \mathrm{feddan}$

$\begin{array}{rrr}207 & 97 & 467 \\ 35 & 17 & 497 \\ 18 & 11 & 607 \\ 6 & 4 & 666 \\ 27 & 21 & 782\end{array}$

293

150

512

* La culture du blé est entièrement irriguée ; dans l'ensemble les rendements sont faibles et les importations augmentent. Cette culture décrốt régulièrement. 